\title{
Results of Deep Brain Stimulation for Parkinson's Disease after 30 Cases
}

\author{
Amit K Ghosh ${ }^{1}$, Aditya Mantry ${ }^{2}$, Sutirtha Hazra ${ }^{3}$, Nilay Shah ${ }^{4}$
}

\begin{abstract}
Introduction: Deep brain stimulation of the subthalamic nucleus (STN) is an effective therapy for medically refractory Parkinson's disease leading to significant improvement of Parkinsonian symptoms through functional inhibition of the STN.

Aim of this study: To analyze the outcome of bilateral subthalamic nucleus, deep brain stimulation in advanced Parkinson's disease patients. This is a clinical observational study.

Material and methods: This is the result of bilateral subthalamic nucleus-deep brain stimulation (STN-DBS) done in 30 patients for advanced Parkinson's disease in the Institute of Neurosciences, Kolkata, during the past 7 years (2013 to 2019, August) by the authors team. Outcome had been analyzed.

Results: Excellent outcome was found after the required programming. Ninety percent patients have shown excellent result. The dosage of antiparkinsonian medications was significantly reduced, with a consequent reduction of dyskinesias.

Conclusion: The effect of the STN-DBS on the motor fluctuations and on the levodopa-induced dyskinesias led to a significant improvement of motor part of Unified Parkinson Disease Rating Scale (UPDRS [III]) rating.

Keywords: Deep brain stimulation, Parkinson's disease, Subthalamic nucleus-deep brain stimulation.

Bengal Physician Journal (2019): 10.5005/jp-journals-10070-6123
\end{abstract}

\section{Objectives}

Hyperactivity of subthalamic nucleus (STN) plays an important role in the pathophysiology of Parkinson's disease. Through chronic high-frequency electrical stimulation, it is possible to achieve functional inhibition of STN leading to improvement of Parkinsonian symptoms and significant reduction of dopaminergic drugs with an improvement of drug-induced dyskinesia. ${ }^{1-5}$

The aim of this study was to analyze the outcome of the bilateral subthalamic nucleus-deep brain stimulation (STN-DBS) for Parkinson's disease during 2013 to 2019 in the Institute of Neurosciences, Kolkata, by the authors team.

\section{Materials and Methods}

Total number of patients-30 (advanced Parkinson's disease). Sex-20 males, 10 females.

Age-51-70 years.

Average duration of disease-10 years.

\section{Preoperative UPDRS (Part III)}

Average score-19 (on medicine), 56 (off medicine). Average preoperative levodopa dosage-956 mg/day. Average duration of motor fluctuations -5 years. Average duration of dyskinesia-4 years.

\section{Predominant Symptoms}

Rigidity and hypokinesia-12.

Tremor-8.

Severe dyskinesia-10.

\section{Patient Selection Methods}

There is a 10-point criteria chart which needs to be "YES" for all except points 8 and 9 (Table 1). ${ }^{4}$ $\overline{{ }^{1-4} \text { Department of Neurosurgery, Institute of Neurosciences, Kolkata, }}$ West Bengal, India

Corresponding Author: Amit K Ghosh, Department of Neurosurgery, Institute of Neurosciences, Kolkata, West Bengal, India, Phone: +91 9830151497, e-mail: amitghosh74@yahoo.co.in

How to cite this article: Ghosh AK, Mantry A, Hazra S, et al. Results of Deep Brain Stimulation for Parkinson's Disease after 30 Cases. Bengal Physician Journal 2019;6(3):55-61.

Source of support: Academic Inspiration

Conflict of interest: None

\begin{tabular}{|c|c|c|}
\hline 1 & Age $<75$ years & Yes \\
\hline 2 & Idiopathic PD (no PSP/MSA/CBD/LBD, etc.) & Yes \\
\hline 3 & Levodopa responsive & Yes \\
\hline \multirow[t]{4}{*}{4} & Poor/adverse response to drug & \\
\hline & (a) Increased off period & Yes \\
\hline & (b) Disabling dyskinesia & Yes \\
\hline & (c) Disabling motor fluctuations & Yes \\
\hline 5 & Degree of disability (UPDRS Part III score) $>25$ & Yes \\
\hline 6 & Neuropsychology, MMSE > 24 & Yes \\
\hline 7 & $\begin{array}{l}\text { Levodopa challenge response positive ( } 30 \% \\
\text { improvement in UPDRS after 12-hour off } \\
\text { medication) }\end{array}$ & Yes \\
\hline 8 & Advanced comorbidity & No \\
\hline 9 & Long-term anticoagulation & No \\
\hline 10 & Willing for surgery and programming & Yes \\
\hline
\end{tabular}

PSP, progressive supranuclear palsy; UPDRS, unified Parkinson's disease rating scale; MSA, multiple system atrophy; $\mathrm{CBD}$, corticobasal degeneration; LBD, Lewy body dementia

(O) The Author(s). 2019Open Access This article is distributed under the terms of the Creative Commons Attribution 4.0 International License (https://creativecommons. org/licenses/by-nc/4.0/), which permits unrestricted use, distribution, and non-commercial reproduction in any medium, provided you give appropriate credit to the original author(s) and the source, provide a link to the Creative Commons license, and indicate if changes were made. The Creative Commons Public Domain Dedication waiver (http://creativecommons.org/publicdomain/zero/1.0/) applies to the data made available in this article, unless otherwise stated. 


\section{Surgical Procedure}

No antiparkinsonism medicine was given in the morning after the last previous night dose to see the clinical effects during awake surgery (Figs 1 to 14).

- Preoperative DBS protocol MRI was under general anesthesia (done day before if surgery planned awake, but done on the same day if surgery planned under general anesthesia)

- Fixation of stereotactic frame (we use Leksell frame) under scalp block if surgery planned awake.

- Planning in StealthStation (computer software)-

- Anatomical STN targeting,

- Trajectory planning,

- Selection of entry point on the skull,

- Getting the stereotactic frame settings.

- Burr hole was done at the entry point selected

- Microelectrode recording (MER) to locate the STN

- Microstimulation to see clinical effects and side effects

- Final electrode placement and confirmed by C-arm fluoroscopy

- Same procedure was repeated in opposite side.

- Pacemaker (battery) placement at subclavicular subcutaneous space.

- Impedance check and programming

Antiparkinsonian drugs to be started as early as possible through Ryle's tube. Pacemaker started "ON" after 48 hours in the low setting.

Patients were usually discharged after 7 or 8 days. The next programming was done after 2 weeks periodically according to clinical effects.

Programming parameters:

- Contact selection,

- Intensity of current (voltage),

- Pulse width (microsecond),

- Frequency $(\mathrm{Hz})$,

- Mode of stimulation (monopolar, bipolar, and tripolar).

Postoperative CT scan of brain and preoperative MRI had been merged in StealthStation to see the best contacts and stimulated accordingly.

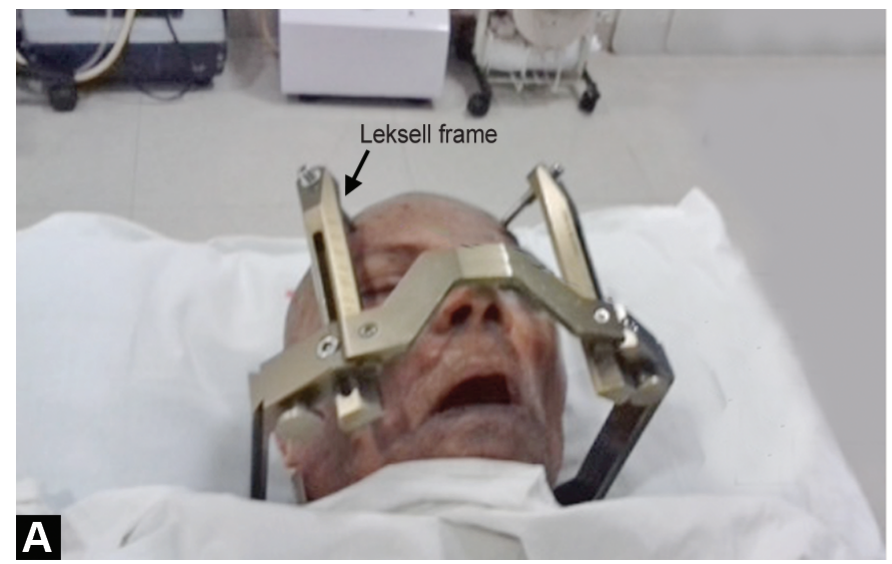

Voltage, pulse width, and frequency had been increased and adjusted according to clinical response.

\section{Results}

Hypokinesia, tremor, rigidity, and dyskinesia of 27 patients had improved significantly.

One patient expired due to neurolept malignant syndrome, followed by pneumonia and septicemia.

One patient developed infection of battery location, required wound debridement, but recovered.

Two patients had small hematoma along the lead track, resolved with conservative treatment. One of them developed hemiparesis.

Twenty-seven patients had bilateral monopolar cathodic stimulation. Two patients had unilateral bipolar stimulation, and one patient had bilateral bipolar stimulation.

Mean stimulation voltage was 2.8 (ranging from 1 to 3 ), pulse width was 60 microsecond (ranging from 60 to 90), and rate ranged from 130 to $180 \mathrm{~Hz}$.

Levodopa was stopped in patients with severe dyskinesia with stimulation $\mathrm{ON}$ and few other antiparkinsonian medications ON (Tables 2 to 4 ).

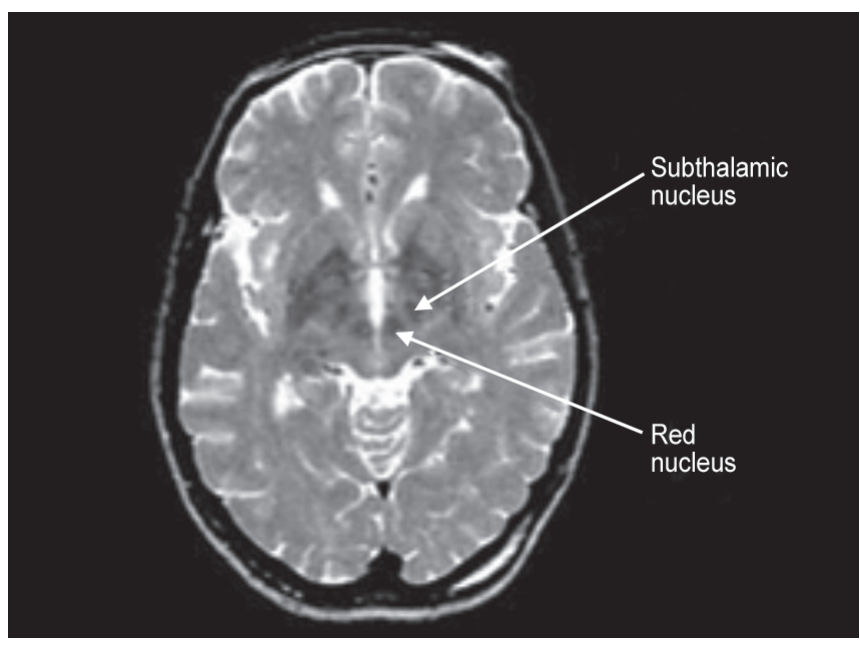

Fig. 2: Location of STN and red nucleus in T2-weighted MRI

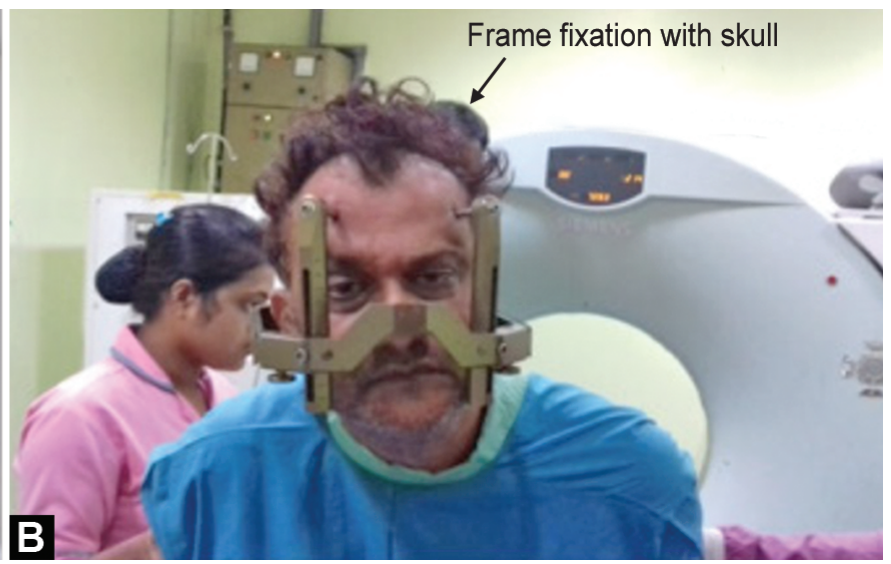

Figs $1 \mathrm{~A}$ and B: (A) Leksell stereotactic frame fixed with skull; (B) CT scan was being done with stereotactic frame 

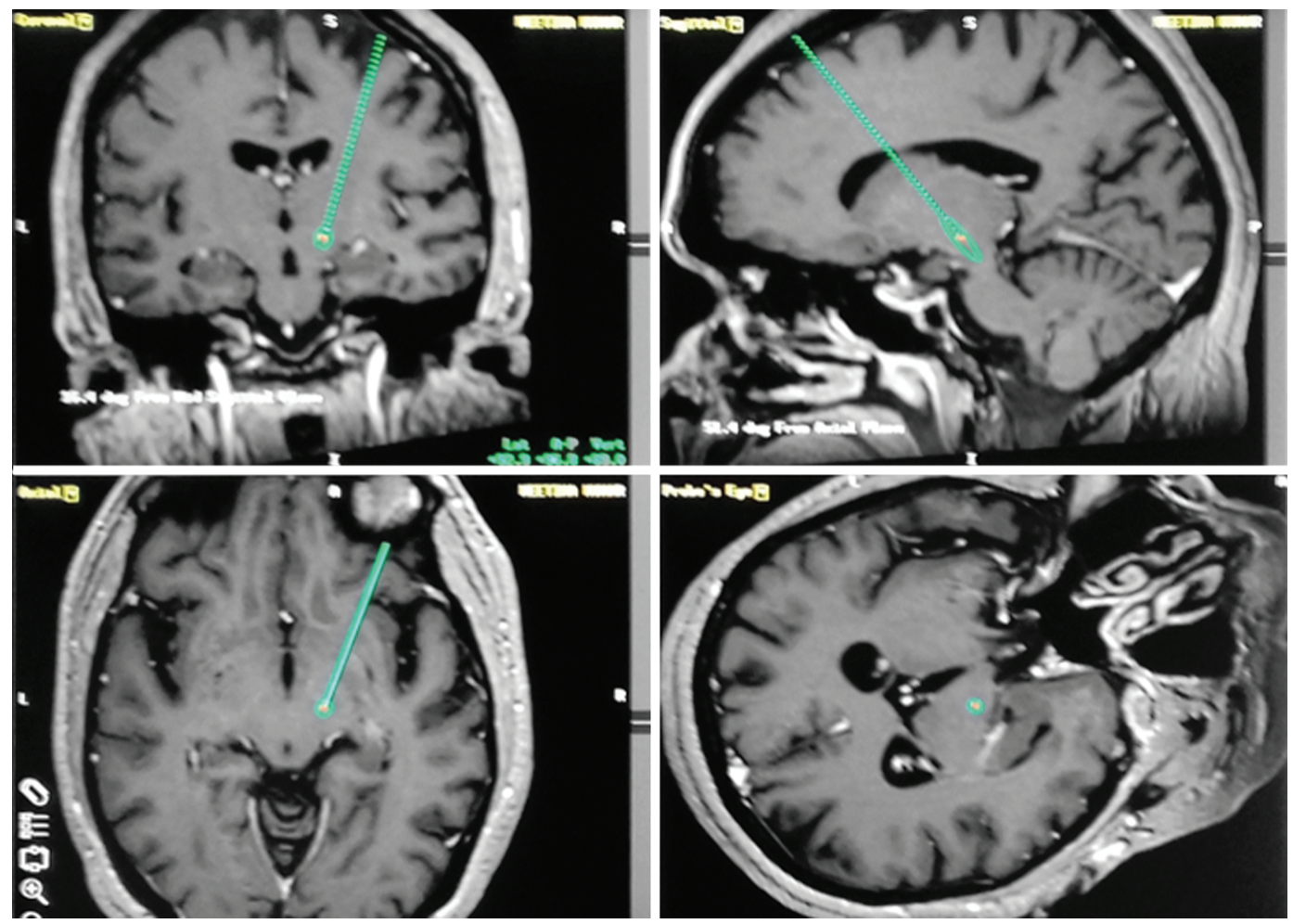

Fig. 3: STN targeting, trajectory and entry point selection at computer planning station from preoperative DBS protocol MRI—right side
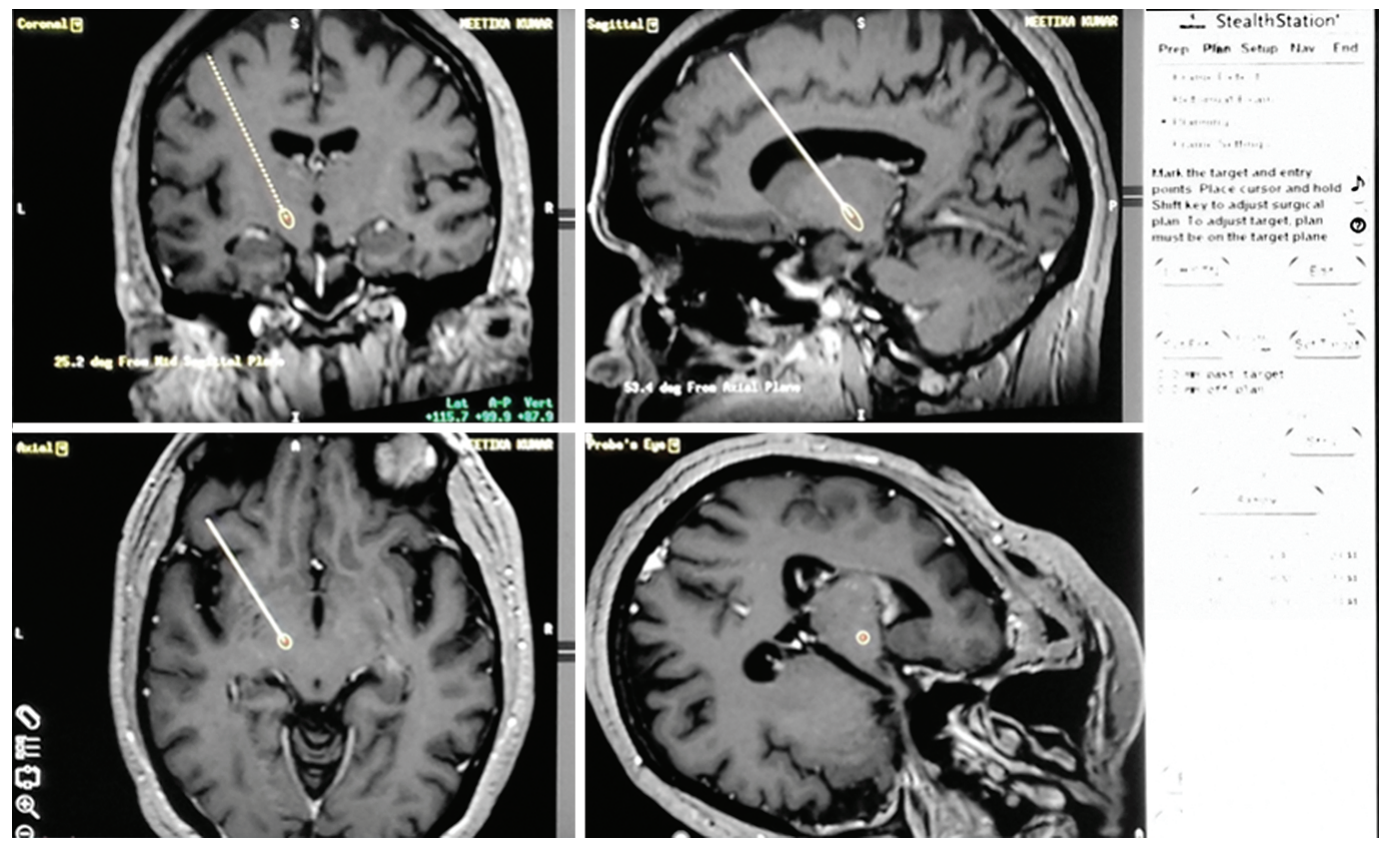

Fig. 4: STN targeting, trajectory and entry point selection at computer planning station from preoperative DBS protocol MRI—left side

\section{CONCLUSION}

The results of STN-DBS in this small series, therefore, seem to be good, effective, and safe for the treatment of select medically refractory Parkinson's disease with a overall $5 \%$ risk of complications, which is comparable to the existing literature.

\section{Acknowledgments}

Dr Hrishikesh Kumar and Dr Purba Basu (Department of Neurology, Movement Disorder), Department of Psychiatry and Psychology, Department of Anaesthesiology and Critical Care, Department of Radiology, Institute of Neurosciences, Kolkata, West Bengal, India. 


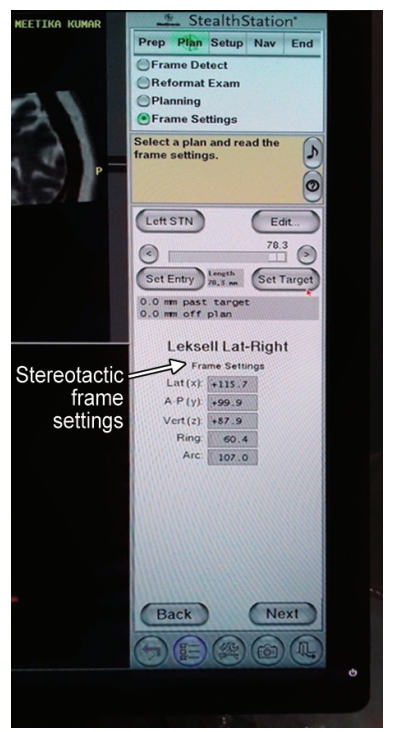

Fig. 5: After selecting target, trajectory and entry point in the computer station based on preoperative MRI, stereotactic coordinates have been generated

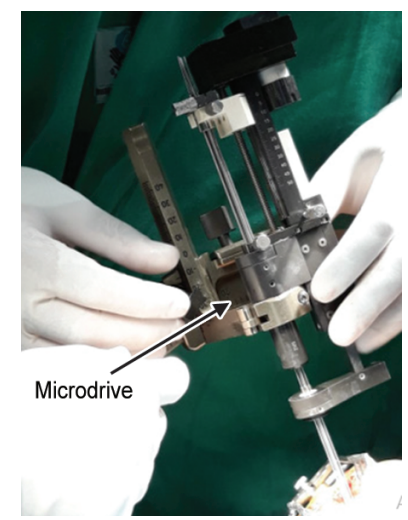

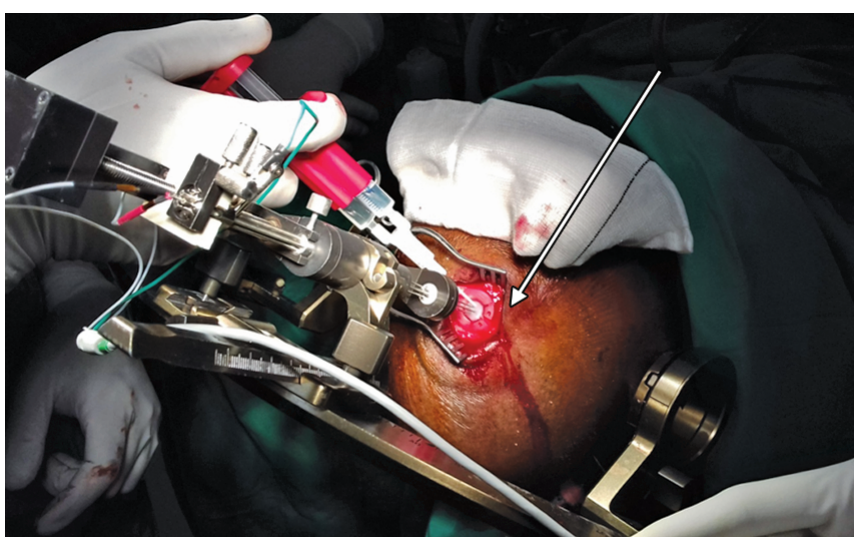

Fig. 6: Burr hole was made at the selected entry point, dura was opened and fibrin glue was given to restrict the CSF egress as CSF egress can cause brain shift resulting malpositioning of lead

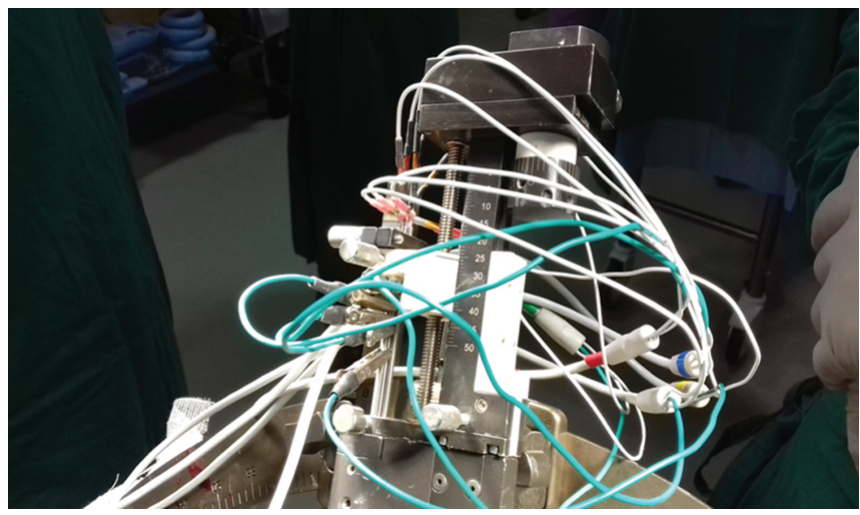

Fig. 8: Microelectrode recording (MER) and microstimulation is going on through microelectrode drive and wires

Fig. 7: Microelectrode drive was attached with stereotactic frame for microelectrode recording

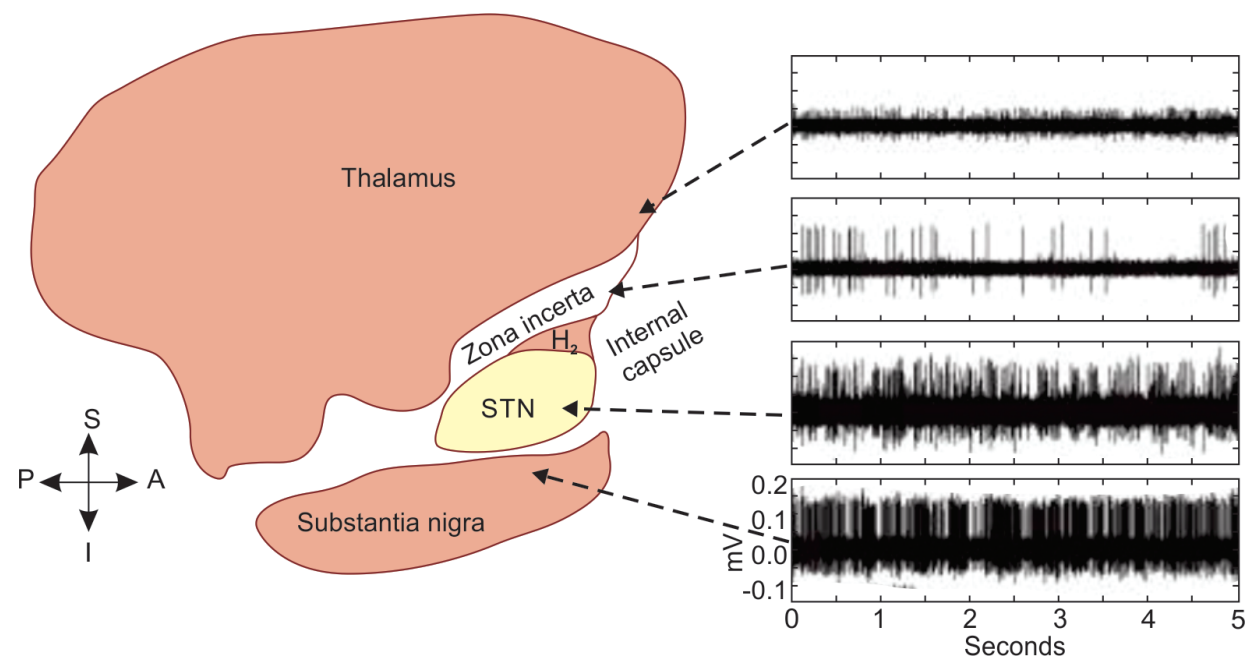

Fig. 9: As the microelectrode (red line) passes through thalamus, zona incerta subthalamic nucleus, and substantia nigra, different electrophysiological graphs will appear, guiding us to know location of the electrode 

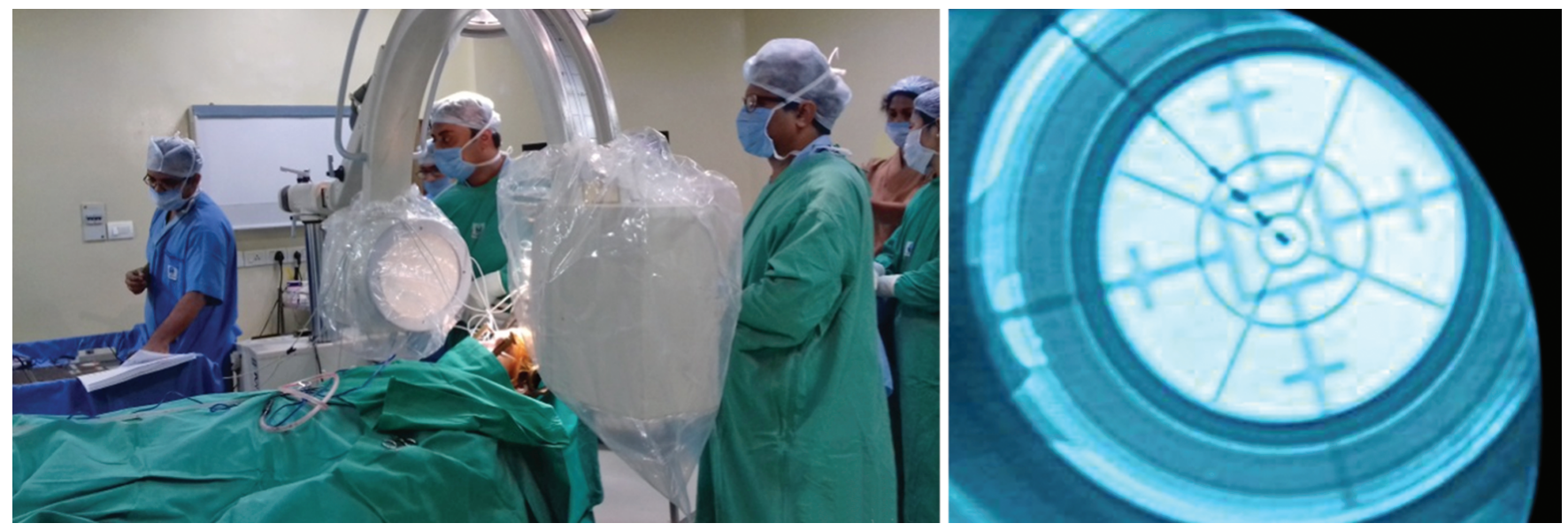

Fig. 10: Intraoperative fluoroscopy to see the lead position

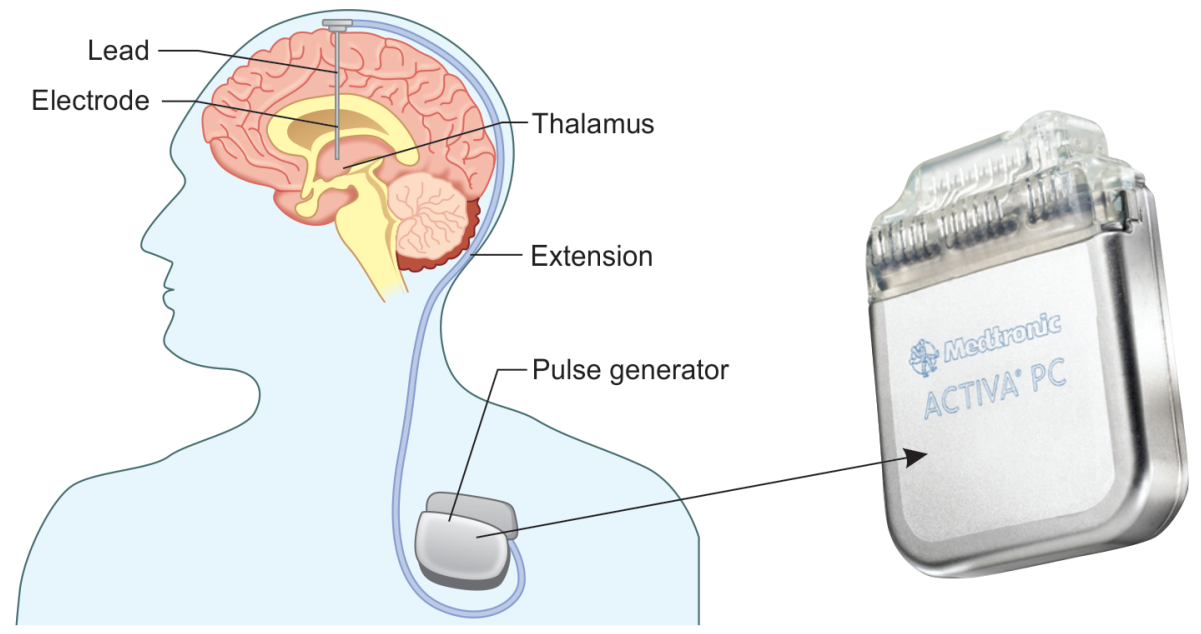

Fig. 11: Components of DBS system (lead, extension wire and pulse generator)

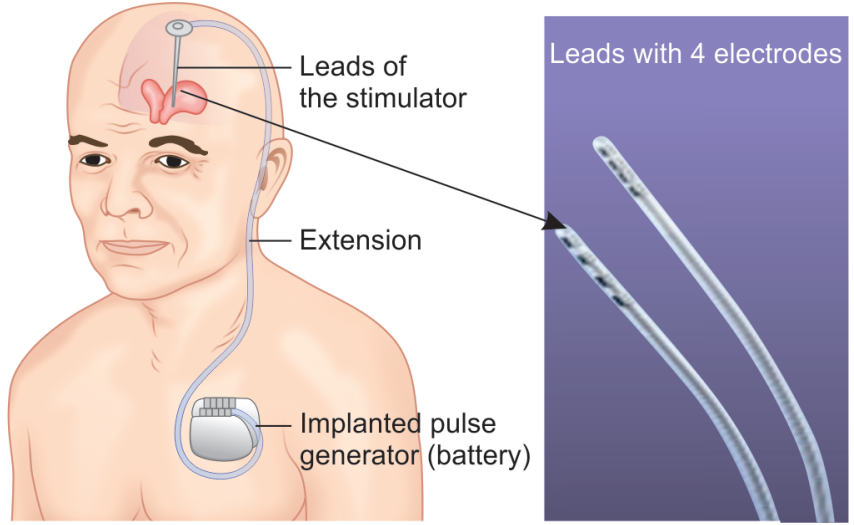

Fig. 12: Leads with four electrodes which remain within STN

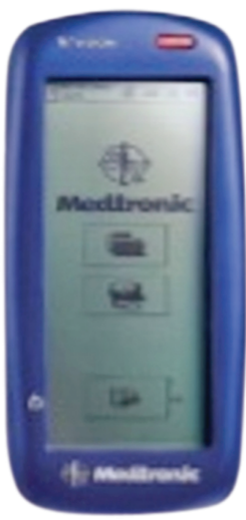

Fig. 13: Programmer for postoperative programming of pacemaker (pulse generator) 


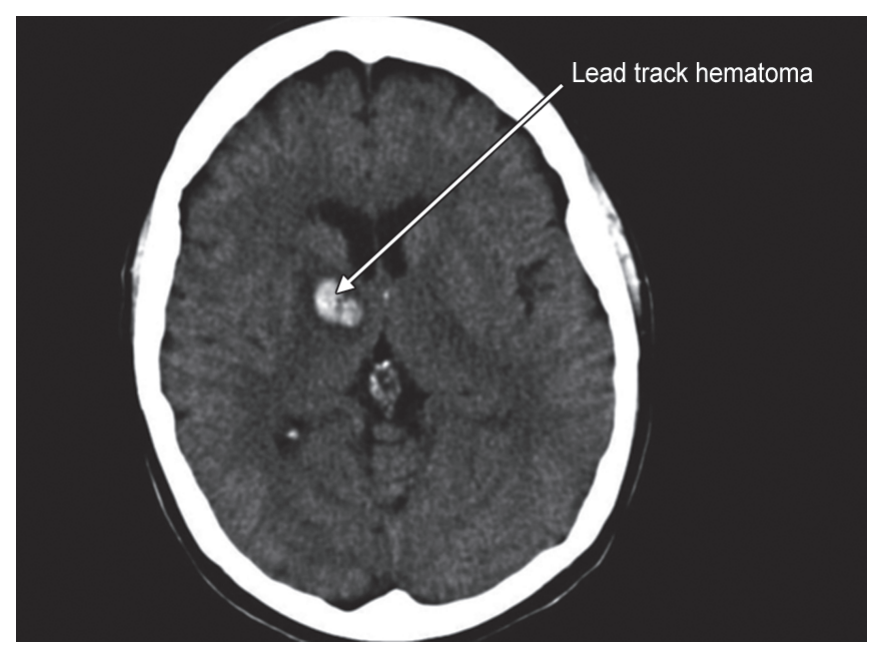

\section{References}

1. Groiss SJ, Wojtecki L, Südmeyer M, et al. Deep brain stimulation in Parkinson's disease. Ther Adv Neurol Disord 2009;2(6):20-28. DOI: 10.1177/1756285609339382.

2. Lopiano L, Rizzone M, Bergamasco B, et al. Deep brain stimulation of the subthalamic nucleus: Clinical effectiveness and safety. Neurology 2001;56(4):552-554. DOI: 10.1212/WNL.56.4.552.

3. Ghosh AK, Hazra S. Deep brain stimulation in Parkinson's disease. Tech Neurosurg Neurol 2019;2(45):TNN.000548. DOI: 10.31031/ TNN.2019.02.000548.

4. Ghosh AK. Patient selection for deep brain stimulation for Parkinson's disease: a very convenient tool. Open Access J Neurol Neurosurg 2019;10(5):555796. DOI: 10.19080/OAJNN.2019.10.555796.

5. Rezai AR, Machado AG, Deogaonkar M, et al. Surgery for movement disorders. Neurosurgery 2008;62(Suppl 2):809-838; discussion 838-9. DOI: $10.1227 / 01$. neu.0000316285.52865.53.

Fig. 14: Postoperative CT scan showing small hemorrhage as complication

Table 2: Comparison between the preoperative and postoperative conditions (mean values)

\begin{tabular}{lcccc}
\hline & \multicolumn{2}{c}{ Before surgery } & \multicolumn{2}{c}{ Stimulation ON } \\
\cline { 2 - 5 } Test & Medication OFF & Medication ON & Medication OFF & Medication ON \\
\hline UPDRS part III (overall average) & 59 & 20 & 25 & 14 \\
Rigidity (item 22) & 4 & 2 & 2 & 1 \\
Akinesia (item 31) & 4 & 2 & 1 & 1 \\
Tremor (items 20 and 21) & 8 & 4 & 1 & 1 \\
Postural stability (item 29) & 3 & 2 & 1 & 0.8 \\
Gait (item 30) & 3 & 1 & 1 & $0 \rightarrow 1$ \\
Speech (item 18) & 2 & 1 & 1 & 2 (levodopa OFF) \\
Part IV dyskinesia (LID) & & 11 (levodopa ON) & & 15 \\
Stand-walk-sit test & & & 20 & 27 \\
Seconds & 48 & 17 & 34 & 0 \\
No. of steps & 73 & 30 & 0 & \\
Clinical fluctuations (items 36, 37, 38, and 39) & & 4 & & \\
\hline
\end{tabular}

Table 3: Clinical outcome after deep brain stimulation from different studies ${ }^{1}$

\begin{tabular}{lcclll}
\hline Studies & No. of patients & Follow-up (years) & $\begin{array}{l}\text { Improvement in } \\
\text { UPDRS III (\%) }\end{array}$ & $\begin{array}{l}\text { Decrease in } \\
\text { OFF time (\%) }\end{array}$ & $\begin{array}{l}\text { Increase in ON time } \\
\text { without dyskinesia }\end{array}$ \\
\hline Krack et al. (1997) & 15 & 1 & 71 & & 200 \\
Kumar et al. (1998) & 7 & 1 & 58 & 72.7 & $270-229$ \\
Limousin et al. (1998) & 20 & 1 & 60 & 61 & \\
DBSPGSG (2001) & 96 & 0.5 & 51 & 61 & $260-265$ \\
Volkmann et al. (2001) & 16 & 1 & 67 & & 192 \\
Pahwa et al. (2003) & 19 & 2.3 & 28 & $56-43$ & 237 \\
Krack et al. (2003) & 49 & 5 & $66-54$ & 64 & 171 \\
Rodriguez-Oroz et al. (2005) & 49 & 3 & $50-39$ & 42 & \\
Fraix et al. (2006) & 95 & 1 & 57 & & \\
Deuschl et al. (2006) & 156 & 0.5 & 41 & 68.2 & \\
Weaver et al. (2009) & 255 & 0.5 & 29 & & \\
Hamani et al. (2005) & 471 & 5 & $56-49$ & 52 & \\
Kleiner-Fisman et al. (2006) & 921 & $>0.5$ & 75 & & \\
Our study (Ghosh et al. (2020)) & 30 & 0.5 & & & \\
\hline
\end{tabular}


Table 4: Complication after DBS related to surgery and hardware according to various studies ${ }^{1}$

\begin{tabular}{|c|c|c|c|c|c|}
\hline Studies & No. of leads & Follow-up (months) & Hemorrhage (\%) & Infection (\%) & $\begin{array}{l}\text { Hardware } \\
\text { complication (\%) }\end{array}$ \\
\hline Binder et al. (2003) & 357 & 60 & 3.1 & & \\
\hline Temel et al. (2004) & 178 & 60 & & 3.8 & \\
\hline Blomstedt and Hariz et al. (2005) & 161 & 40 & & 3 & 17.3 \\
\hline Deuschl et al. (2006) & 156 & 6 & 1.9 & 3.8 & 1.3 \\
\hline Goodman et al. (2006) & 181 & 4 & 2 & 4.7 & 11.5 \\
\hline Voges et al. (2006) & 352 & 56 & 0.2 & 5.7 & 13.9 \\
\hline Seijo et al. (2007) & 252 & 37 & 6.9 & & 3.84 \\
\hline Kenney et al. (2007) & 507 & 10 & 1.5 & 4.4 & 4 \\
\hline Tir et al. (2007) & 206 & 1 & 5.8 & 6.8 & 3.9 \\
\hline Sillay et al. (2008) & 759 & 6 & & & 4.5 \\
\hline Weaver et al. (2009) & 242 & 6 & 0.8 & 9.9 & 6.6 \\
\hline Hamani et al. (2005) & 471 & & 2 & & 9 \\
\hline Hamani and Lozano (2006) & 922 & & 2.8 & 6.1 & 11.4 \\
\hline Kleiner-Fishman et al. (2006) & 921 & & 3.9 & 3.6 & 4.5 \\
\hline Videnovic and Metman (2008) & 2205 & & 3.8 & 2.9 & 5 \\
\hline Our study (Ghosh et al. (2020)) & 60 & 6 & 3.3 (2 patients) & 1 (battery location) & 1 (kinking of wires) \\
\hline
\end{tabular}

